\title{
Prospective assessment of Quality of life in Ovarian cancer survivors
}

Chinna Babu Dracham ${ }^{1}$, Renu Madan ${ }^{1}$, Arun Elangovan ${ }^{1}$, Bhavana Rai ${ }^{1}$, Sushmita Ghoshal ${ }^{1}$

${ }^{1}$ Post Graduate Institute of Medical Education and Research (PGIMER), Radiotherapy and Oncology, Chandigarh, India.

\section{BACKGROUND}

- Ovarian cancer treatment consists of extensive surgery with multi-drug chemotherapy and such intensive treatment is associated with significant morbidity.

- As there is diversity in ovarian cancer survivorship, addressing the issue of Quality-of-life (QoL) is becoming important for themselves and their families.

-QoL has been used as an endpoint in clinical trials to identify the consequences of cancer treatment, assessment of rehabilitation needs and predict response to future treatment.

-Our goal was to assess the QoL in long-term ovarian cancer survivors and to identify the factors related to poor QoL.

\section{AIMS OF THE STUDY}

1) To assess the Quality of Life in long term ovarian cancer survivors

2) To identify the factors associated with poor quality of life

\section{MATERIAL AND METHODS}

Ovarian cancer patients, diagnosed at-least two years back from the date of recruitment were included in the study after obtaining clearance from Institute ethics committee.

Inclusion criteria:

- Ovarian cancer patients of stage I-IVA (FIGO-2009) with histological proof of malignancy

- Patients who have the ability to understand the study procedure and to give the informed consent

Exclusion criteria:

-Residual/ recurrent disease

-Wide spread metastatic disease at initial presentation (Stage IVB)

-Previous history of malignancy other than ovarian primary

\section{RESULTS}

The study was conducted over a period of eight months, from April 2017 to November 2017. The median age was 45-years (range; 17-72).

Global QoL scores varied with age $(\mathrm{p}=0.019)$ and number of chemotherapy cycles $(\mathrm{p}=0.007)$. Clinical parameters like age, stage, comorbidities, number of chemotherapy cycles and drugs were the most consistent factors affecting functional and symptomatic scales.
Table 1: Disease characteristics Disease characteristics

No. of patients (\%)

Baseline CA 125: mean

Stage:

I

II

III

IV

Hstology:

Germ cell tumors

Sex cord stromal tumors

Epithelial

2284.7 (range; $10.4-57900)$

Recurrence status:

No recurrence

Once recurrence

$42(50 \%)$

$2(2.4 \%)$

$36(42.9 \%)$

$4(4.8 \%)$

$6(7.1 \%)$

$6(7.1 \%)$

$72(85.8 \%)$

Twice recurrence

$52(61.9 \%)$

$26(31 \%)$

$6(7.1 \%)$

CA 125 at last follow-up: mean

21 (range; 4-48)

Table 2: Treatment characteristics

Treatment characteristics

No. of patients (\%)

Median follow-up

47.5 months (range: $24-169$ )

Treatment:

a. Surgery alone

$14(16.7 \%)$

b. Surgery + chemotherapy

c. Surgery + chemotherapy +

$60(71.4 \%)$

$10(11.9 \%)$

radiotherapy

Platinum free interval $(\mathrm{N}=\mathbf{2 8})$

Median: 18.5 months (range; 2-87)

\section{CONCLUSION}

In this era of prolonged survival in ovarian cancer patients, evaluation of QoL in survivors is important to address unmet needs and to improve physical, social, emotional and sexual well-being.

Comprehensive supportive care services should be provided whenever necessary.

Table 3. Univariate analysis (ANOVA) of clinic-pathological characteristics with QOL score

\begin{tabular}{|c|c|c|c|c|c|c|c|}
\hline QOL measures & $\mathbf{N}$ & Median score (IQR) & Age & Co-morbidities & Stage & Number of chemotherapy cycles & Number of chemotherapy drugs \\
\hline \multicolumn{8}{|l|}{ QOQ-C30 } \\
\hline Global QOL & 84 & $83.3(66.6-100)$ & 0.019 & & & 0.007 & \\
\hline Physical functioning & 84 & 90(73.3-100) & 0.031 & & & & \\
\hline Role of functioning & 84 & $100(100-100)$ & & 0.001 & & & \\
\hline Social functioning & 84 & $100(83.3-100)$ & 0.037 & & 0.004 & 0.001 & 0.001 \\
\hline \multicolumn{8}{|l|}{ Symptom scales } \\
\hline Fatigue & 84 & 22.2(11.1-33.3) & 0.008 & 0.054 & & & \\
\hline Nausea \& vomiting & 84 & $0(0-16.6)$ & & & & 0.035 & \\
\hline
\end{tabular}

